\title{
(6) OPEN ACCESS \\ Tumor histopathological response to neoadjuvant chemotherapy in childhood solid malignancies: is it still impressive?
}

\author{
Ehab Hanafy, Abdullah Al Jabri, Gelan Gadelkarim, Abdulaziz Dasaq, \\ Faisal Nazim, Mohammed Al Pakrah
}

Prince Sultan Oncology Center, King Salman Armed Forces Hospital, Tabuk, Saudi Arabia

\section{Correspondence to} Dr Ehab Hanafy, Prince Sultan Oncology Center, King Salman Armed Forces Hospital, Tabuk 71411, Saudi Arabia;

ehab.hmahmoud@gmail. com

Accepted 28 August 2017

Published Online First

27 September 2017
Check for updates

To cite: Hanafy $\mathrm{E}, \mathrm{Al}$ Jabri A, Gadelkarim G, et al. J Investig Med 2018;66:289-297.

\section{ABSTRACT}

The management of oncological malignancies has significantly improved over the last decades. In modern medicine, new concepts and trends have emerged paving the way for the era of personalized and evidence-based strategies adapted to the patients' prognostic variables and requirements. Several challenges do exist that are encountered during the management, including the difficulty to assess chemotherapy response with certainty. Having known that neoadjuvant chemotherapy might be the only solution for a proportion of patients with tumors that are unresectable at diagnosis, emergence of strategies that use risk group-directed therapy became an integral part in the management of oncological malignancies. Tumor histopathological change post neoadjuvant chemotherapy is one of the most important predictors of management outcome and is being used in many chemotherapy protocols as an essential determinant of the most suitable postoperative chemotherapy regimen. Bone tumors are the classic models of this approach; however, other childhood solid tumors show significant variations in outcome as a result of tumor histopathological response to neoadjuvant chemotherapy. The aim of this review is therefore to summarize the significance of histopathological responses seen after neoadjuvant chemotherapy in childhood solid tumors. Moreover, it suggests that the effect on tumor histopathology through modifying neoadjuvant chemotherapy and, on the other hand, toxicities from intensifying adjuvant chemotherapy might either necessitate the change of a number of arm groups in different protocol regimens or include newer chemotherapeutic agents adjuvantly for better outcome and lesser toxicities in poor tumor histopathological responders.

\section{INTRODUCTION}

Tumor necrosis and histopathological changes in childhood solid tumors following neoadjuvant chemotherapy are important predictors of disease outcome. Survival rates are better in good responders to neoadjuvant chemotherapy with high tumor necrosis than those with poor response. Despite these facts, there is little evidence to recommend for more intensified adjuvant chemotherapy regimens for poor responders as no benefits are seen with regard to disease outcome when compared with historical counterparts. These can be seen clearly from results like in large osteosarcoma trials where ifosfamide and etoposide (IE) did not show a valid benefit when added adjuvantly to highrisk patients and in Wilms' tumor (WT) where doxorubicin can be safely removed from postoperative chemotherapy in intermediate-risk patients; even the results have to be validated in Ewing's sarcoma (ES) despite the promising results in the busulfan/melphalan arm in poor responders.

A widely used method for histological assessment was described by Huvos and was based on evaluation of osteosarcoma samples. ${ }^{1}$ The Huvos system includes grades I-IV according to the degree of necrosis (table 1). ${ }^{2}$

This method showed effectiveness in the management of ES as the degree of necrosis has been directly correlated with the improvement of overall survival (OS). ${ }^{2}$ There is a difference between ES and osteosarcoma as ES does not produce any major extracellular matrix component, so there is no evidence left by the tumor cells. Furthermore, ES cells may disappear completely in response to chemotherapy. Due to this fact, there might be a decrease in tumor volume after neoadjuvant chemotherapy without histological delineation of where the tumor was located originally. ${ }^{3}$ Dramatic changes in tumor volume following neoadjuvant chemotherapy make it difficult to calculate tumor necrosis based only on the viable cells per unit area of residual tumor. So, a quantitative way to calculate tumor necrosis is not optimal in ES. ${ }^{4}$

Picci et al proposed evaluation of the amount of remaining viable tumor rather than the amount of non-viable tumor. The scoring system proposed by Picci et al includes three grades. ${ }^{4}$ Grade 1 represents a tumor with at least one macroscopic residual nodule of viable tumor. Grade 2 represents a tumor with only isolated microscopic foci of viable tumor. Grade 3 indicates no evidence of viable tumor cells. ${ }^{3}$ This method is easy to interpret; however, it fails to determine the original size of the tumor as seen when the persistence of one nodule is 
Table 1 Schemes for assessment of tumor responsiveness in osteosarcoma and Ewing's sarcoma ${ }^{2}$

\begin{tabular}{llll}
\hline Description & Huvos & $\begin{array}{l}\text { Salzer- } \\
\text { Kuntschik }\end{array}$ & WHO 2013 \\
\hline $\begin{array}{l}\text { No vital tumor cells } \\
\text { Single vital tumor cells or one vital }\end{array}$ & IV & I & Responder \\
tumor nest smaller than $0.5 \mathrm{~cm}$ & & II & \\
Less than $10 \%$ vital tumor tissue & III & III & \\
$10-50 \%$ vital tumor tissue & II & IV & Non- \\
More than $50 \%$ vital tumor tissue & - & V & responder \\
No effect of chemotherapy & I & VI & \\
\hline
\end{tabular}

graded the same, regardless of whether the tumor volume was $10 \mathrm{~cm}^{3}$ or $200 \mathrm{~cm}^{3}$.

\section{REVIEW OF MOST COMMON SOLID TUMORS OSTEOSARCOMA}

The extent of tumor necrosis after neoadjuvant chemotherapy is the most important predictor of outcome in patients with osteosarcoma. Different degrees of necrosis on histological examination of tumor specimens were first noted by investigators from Memorial Sloan-Kettering Cancer Center (MSKCC) and it was observed that significant necrosis is associated with better event-free survival (EFS) and OS. ${ }^{6-8}$

Good responders are those who have tumor necrosis of more than $90 \%$ corresponding to grades III and IV, whereas poor responders are those who have tumor necrosis of less than $90 \%$ corresponding to grades I and II. ${ }^{9}{ }^{10}$ The diseasefree survival rates are $50 \%$ and $80 \%$, respectively (figure 1), (table 2).

Different osteosarcoma protocols have used the poor response to neoadjuvant chemotherapy as a determinant of more intensified adjuvant chemotherapy. Protocols used by Children's Cancer Group (COG), Rizzoli Institute, and German-Austrian-Swiss Cooperative Osteosarcoma Study Group (COSS) include neoadjuvant chemotherapy consisting of high-dose methotrexate (HDMTX) and bleomycin + cyclophosphamide + dactinomycin (BCD) and adjuvant chemotherapy was intensified with the addition

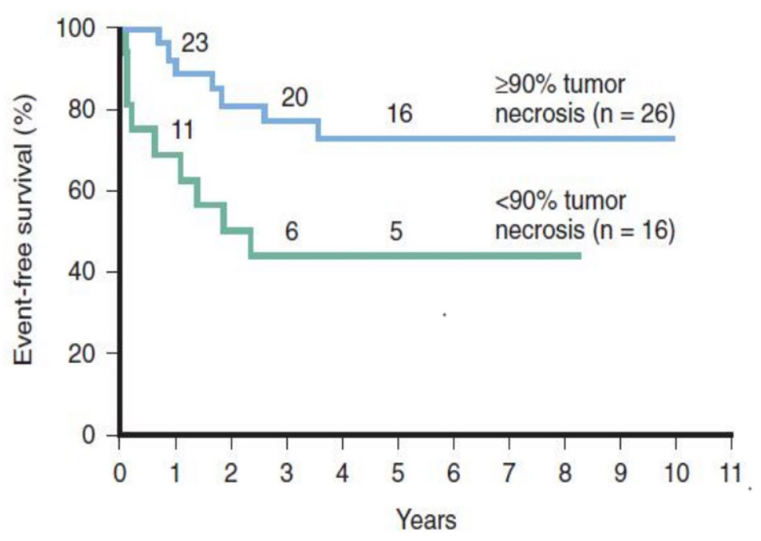

Figure 1 Event-free survival of patients with localized osteosarcoma who had $\geq 90 \%$ or $<90 \%$ tumor necrosis after neoadjuvant chemotherapy. ${ }^{89}$
Table 2 Grading of histological response to neoadjuvant chemotherapy in osteosarcoma ${ }^{910}$

\begin{tabular}{lll}
\hline $\begin{array}{l}\text { Grading of histological response to neoadjuvant chemotherapy in } \\
\text { osteosarcoma }\end{array}$ \\
\hline Response & Grade & Histology \\
\hline Poor & I & $\begin{array}{l}\text { Necrosis minimal or absent } \\
\text { Necrosis is }<90 \% \text { of the tumor but greater than } \\
\text { minimal }\end{array}$ \\
Good & III & $\begin{array}{l}\text { Scattered areas of viable tumor but }>90 \% \text { of tumor } \\
\text { necrotic } \\
\text { No viable tumor }\end{array}$ \\
\hline
\end{tabular}

of cisplatin (CP) and doxorubicin. ${ }^{11} 12$ Neoadjuvant treatment consisted of HDMTX, BCD, and doxorubicin in the MSKCC T10 protocol and CP was the drug used to intensify the adjuvant phase. Patients in the poor responder group had a similar outcome to those who did not receive augmented chemotherapy, in spite of chemotherapy intensification except in T10 protocol.

Similarly, HDMTX, doxorubicin, and CP were given in the neoadjuvant phase in the Scandinavian Sarcoma Group (SSG) protocol SSGVIII and recent Rizzoli trial, ${ }^{13} 14$ so new drugs like IE were offered as adjuvant chemotherapy to poor responders. In the Rizzoli trial, patients did not have a better outcome than those who did not receive IE intensification, whereas in the SSGVIII trial, the outcome was better, with an OS of 70\% in patients with a poor histological response. ${ }^{8}$

Using another approach, COSS stratified patients into high-risk and low-risk groups based on pathology, tumor size, and clinical response to neoadjuvant therapy. Patients in the high-risk group were treated with HDMTX, doxorubicin, and $\mathrm{CP}$ in addition to ifosfamide. The stratification has not succeeded as the outcome of the high-risk group was the same as the low-risk one. ${ }^{8}$ On the other hand, there is a good evidence that attempting to reduce toxicity for low-risk patients who have a good chemotherapy response by minimizing chemotherapy results in a worse prognosis. ${ }^{11} 12$

A large European and American Osteosarcoma Study Group-1 (EURAMOS-1) trial that represents a collaboration between four research groups in osteosarcoma, the COG, COSS, European Osteosarcoma Intergroup (EOI) and the SSG investigated whether more intensive adjuvant chemotherapy to poor responders with high grade osteosarcoma (more than 10\% viable tumor) improved EFS. Patients were randomised to receive postoperative methotrexate, cisplatin, and doxorubicin (MAP) or MAP plus ifosfamide and etoposide (MAPIE). Because of increased toxicity, secondary leukemias and no improvement in EFS, EURAMOS-1 results recommended against any adjustment to the standard of care and against the addition of IE to adjuvant chemotherapy in poorly responding osteosarcoma (figure 2, figure 3 , figure 4 ). ${ }^{15}$

All data considered, there seems to be no solid evidence that intensifying adjuvant chemotherapy for patients with osteosarcoma with poor histological response led to an improved outcome. 


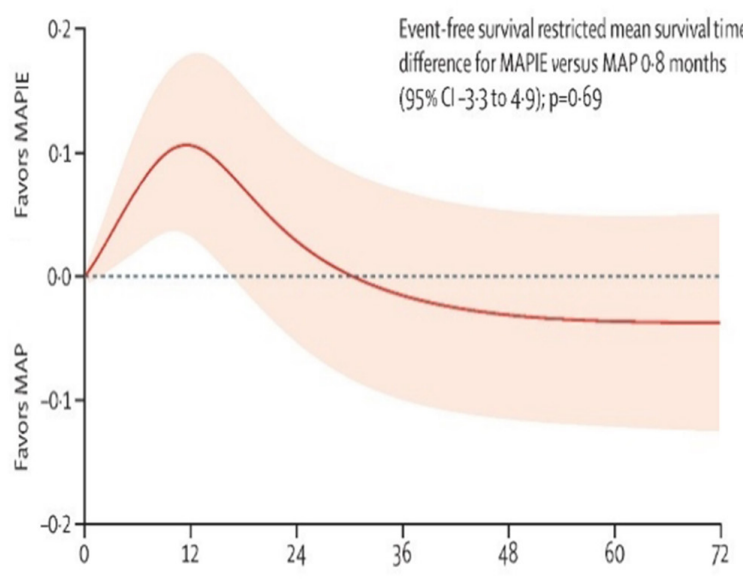

Figure 2 Absolute difference in event-free survival by flexible parametric model difference. $95 \% \mathrm{Cl}$ is shown by shading. ${ }^{15}$ MAP, methotrexate, cisplatin, and doxorubicin; MAPIE, MAP plus ifosfamide and etoposide.

\section{EWING'S SARCOMA (ES)}

With the same concept of osteosarcoma, different authorities used the histopathological response to neoadjuvant therapy as a predictor of outcome. Patients with more than $90 \%$ tumor necrosis post neoadjuvant chemotherapy have a reduced risk of disease recurrence compared with patients who have less necrosis. Moreover, in patients with non-metastatic tumors of extremities, chemotherapy response is prognostic independent of age and tumor size. Despite these results, grading of chemotherapy response in ES has not yet become routine practice. ${ }^{8}$

In one study by Wunder et al, the histological response to chemotherapy was graded semiquantitatively. Grade I for necrosis of $50 \%$ of the tumor or less; grade II for necrosis of $>50 \%$ but $<90 \%$; grade III for necrosis of $90-99 \%$; and grade IV for necrosis of $100 \%$ of the tumor. The histological response to preoperative chemotherapy, followed by tumor size were the most essential predictors of EFS. The rate of EFS was 0 of 14 patients who had had a grade-I response at 5 years, 6 of 16 patients who had had a grade-II response, and $37(84 \%)$ of 44 patients who had had a grade-III or grade-IV response (figure 5) and accordingly concluded

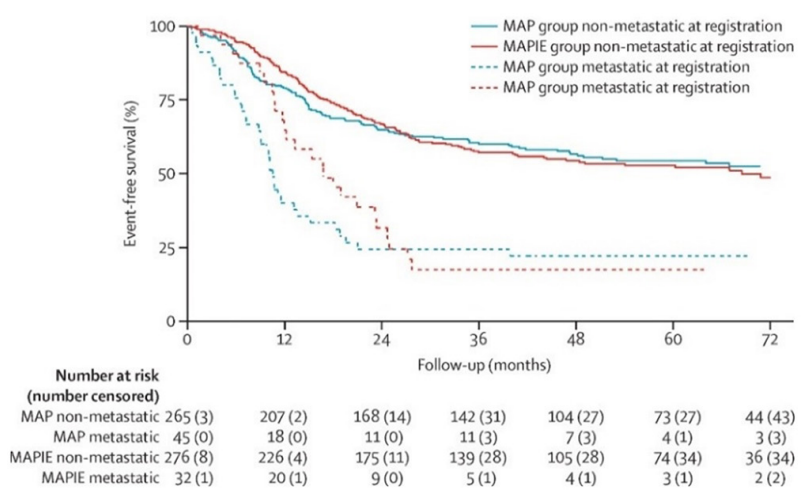

Figure 3 Event-free survival by non-metastatic osteosarcoma status and metastatic osteosarcoma status at registration. ${ }^{15}$ MAP, methotrexate, cisplatin, and doxorubicin; MAPIE, MAP plus ifosfamide and etoposide.

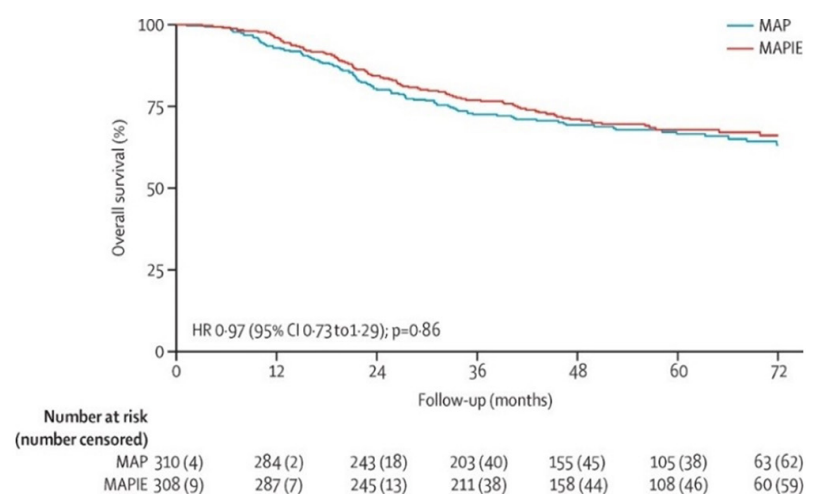

Figure 4 Overall survival in osteosarcoma. ${ }^{15} \mathrm{MAP}$, methotrexate, cisplatin, and doxorubicin; MAPIE, MAP plus ifosfamide and etoposide.

that these prognostic factors should be used to identify a group of patients who are at a high risk for distant metastasis as intensive or novel therapies can be used to improve their disease outcome. ${ }^{2}$

Lin et al reported that some factors may be associated with local recurrence of ES, patients with a good response to chemotherapy ( $\geq 90 \%$ tumor necrosis), patients who had superior locoregional-free survival at 5 years $(p=0.015)$ and that positive surgical margin was not a strong predictor of recurrence $(p=0.72){ }^{16}$ The relevance of the histological response of the primary tumor to initial chemotherapy both in localized and systemic diseases emphasizes the need for a high intensity induction treatment in patients with ES. ${ }^{17}$

In a meta-analysis of several trials, Smith et al demonstrated that an induction chemotherapy including doxorubicin with each cycle is superior to protocols alternating doxorubicin with actinomycin D. ${ }^{18}$ In European Intergroup Cooperative Ewing Sarcoma Study (EICESS 92), vincristine, dactinomycin, ifosfamide, doxorubicin (VAIA) and etoposide, vincristine, dactinomycin, ifosfamide, doxorubicin (EVAIA) for induction therapy achieved tumor response rates above those obtained with the VACA regimen in the CESS 81 study. All considered, a further increase of treatment intensity during the induction treatment, particularly during the time when the primary tumor is still in place, was considered an option of further improving both the histological response and the survival in patients with ES.

Patients with $>10 \%$ viable tumor cells at surgery following neoadjuvant therapy in CESS/EICESS studies had a less favorable outcome with an EFS of 0.47 after 10 years. Patients with good histological response (less than 10\% viable tumor) after chemotherapy alone had a prognosis of about 0.70 after 10 years.

In a combined analysis, data on patients treated surgically from the CESS/EICESS and the Société Francaise d'Oncologie Pédiatrique Ewing tumor (SFOP EW) studies demonstrated the impact of histological response was more prominent than the impact of tumor volume. By contrast, when radiation therapy is the initial local control, survival is independent of histological response.

Based on data from ET-1, ET-2, EW88, EW93, CESS81, CESS 86, EICESS92, and other European studies, the Ewing tumour Working Initiative of National Groups (EURO EWING 99) (figure 6) stratified the patients according to 


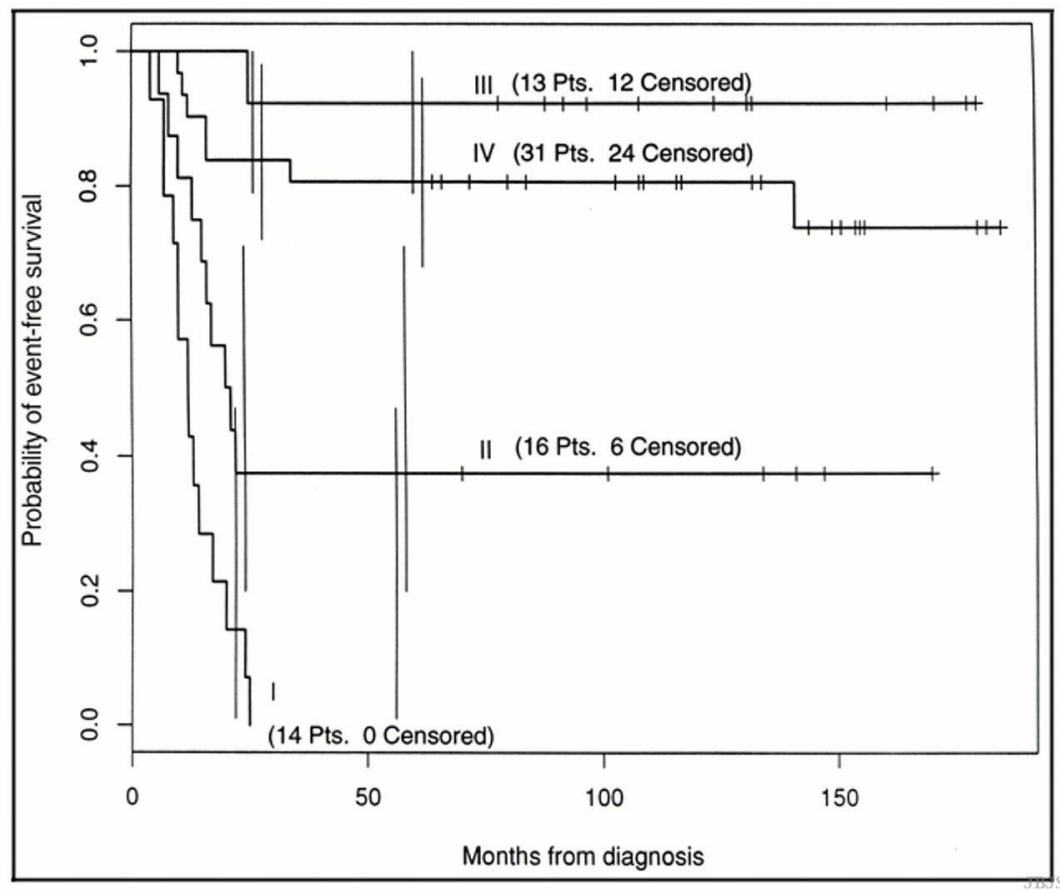

Figure 5 The relationship between event-free survival (EFS) and the histological response of Ewing's sarcoma to neoadjuvant chemotherapy ( $p=0.0001$ ), with 95 per cent Cls. Curve I=no necrosis (grade I), curve II=moderate necrosis (grade II), curve $\mathrm{III}=$ almost complete necrosis (grade III), and curve IV=complete necrosis (grade IV). The tick marks indicate the times of the latest follow-up evaluations. Censored indicates EFS. ${ }^{2}$

metastatic profile, site of metastases in stage IV patients, feasibility of local therapy options and histological response to chemotherapy, and initial tumor volume of $<200 \mathrm{~mL}$ $1 \geq 200 \mathrm{~mL}$ in patients where surgery is not feasible as primary local control.

In patients with high-risk and localized ES, busulfan-melphalan was extremely more potent than VAI as assessed by 3 -year EFS (67\% vs 53\%) and 3-year OS (78\% vs 70\%). Better effect in the busulfan-melphalan arm is attributed primarily to a reduction in risk of metastases, noting a $41 \%$ reduction in the incidence of metastases with busulfan-melphalan over VAI. Whereas in patients with pulmonary metastases, high-dose chemotherapy was not superior to standard chemotherapy, 3-year EFS rates were 55\% with busulfan-melphalan and 51\% with VAI plus whole lung radiation $(\mathrm{p}=0.24) .{ }^{19}$

Having known that utilization of high-dose chemotherapy may be limited and only $45 \%$ of eligible patients with localized disease (216 of 477 ) and $55 \%$ of patients with pulmonary metastases (265 of 480) were enrolled on the trial, consideration regarding busulfan-melphalan as a part of standard care of high-risk patients should be further validated.

Induction chemotherapy with VIDE is now considered the standard of care in ES in Europe, whereas compressed vincristine, doxorubicin, and cyclophosphamide-ifosfamide plus etoposide (VDC-IE) is the North American standard. The results of the ongoing Euro-Ewing 2012 trial (figure 6) comparing these two regimens will define the international standard induction chemotherapy for ES. The possible benefit of zoledronic acid added to conventional maintenance chemotherapy is under evaluation in both
Euro-Ewing 2012 and Ewing 2008 trials (figure 6) that use histological response for further randomization of patients. On the basis of the efficacy of the cyclophosphamide plus topotecan combination observed in recurrent ES, the Children's Oncology Group (COGAEWS1031) trial (figure 6) is currently evaluating the addition of this combination to compressed VDC-IE. ${ }^{20}$

Taking these data into consideration, despite the application of tumor histopathological response to stratify treatment in ES in many protocols, we find no strong evidence that intensifying chemotherapy in poor responders results in better outcome or at least still needs further validation.

\section{WILM'S TUMOR}

Management of WT is based on trials from two large groups: the COG Renal Tumor Committee (COG RTC) and International Society of Pediatric Oncology (SIOP). The COG RTC recommends for an initial nephrectomy if feasible followed by postoperative chemotherapy with or without radiotherapy in a group of patients. ${ }^{21}$

Patients treated on SIOP studies receive preoperative chemotherapy which usually affects necrosis of immature and actively proliferating cells in WT, whereas slowly replicating and differentiated cells are unaffected. The microscopic appearance of the tumor after chemotherapy has prognostic significance. Approximately 5-10\% of WTs are completely necrotic after chemotherapy, a finding associated with a $98 \% 5$-year relapse-free survival rate. ${ }^{22}$ By contrast, WTs with a predominance of blastemal cells after chemotherapy, defined as viable cells in more than one-third of the tumor mass and blastemal cells in at least two-thirds of 


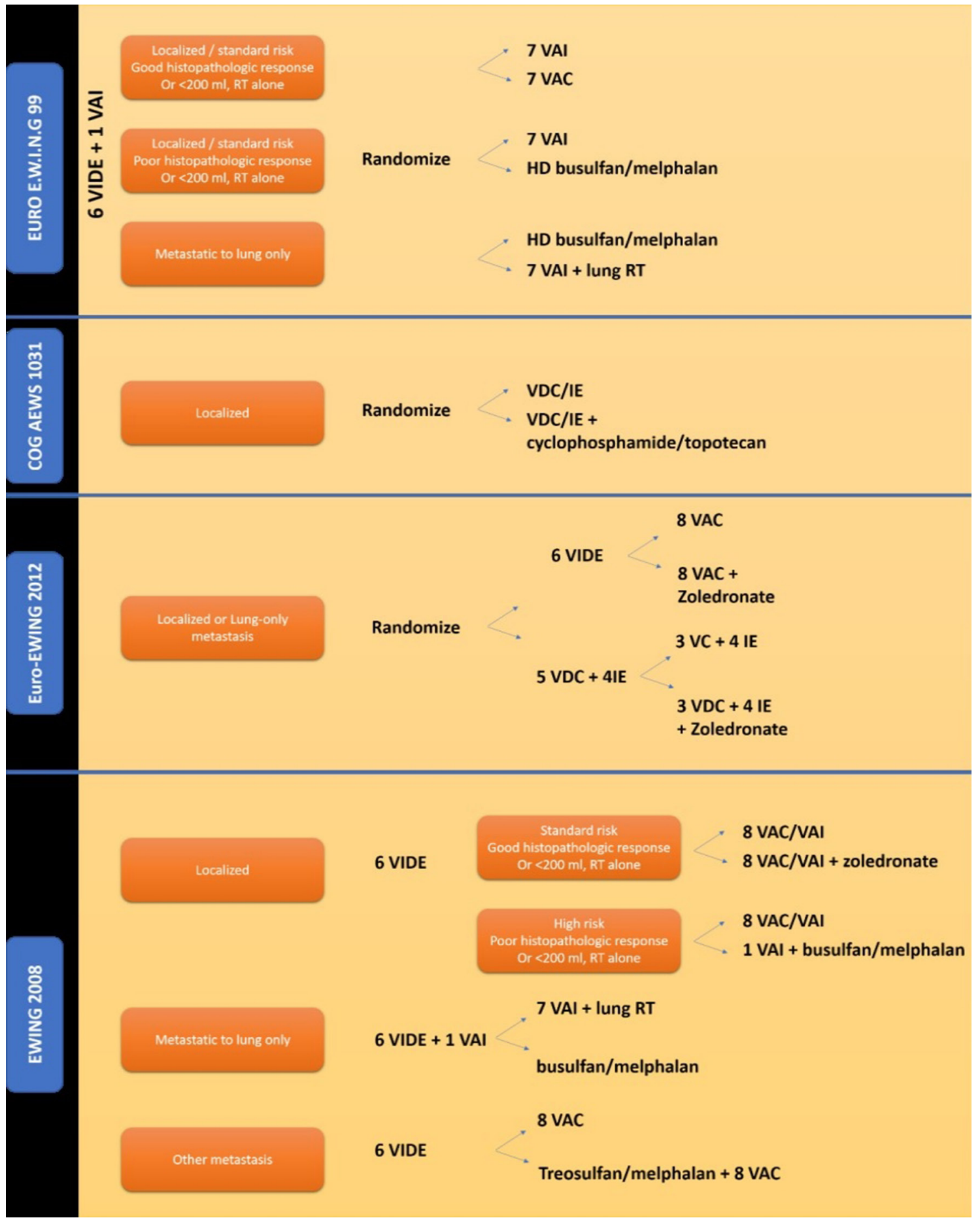

Figure 6 Randomized trials for Ewing's sarcoma. COG, Children's Oncology Group; IE, ifosfamide and etoposide; RT, radiotherapy; VAC, vincristine, dactinomycin, and cyclophosphamide; VAl, vincristine, dactinomycin, and ifosfamide; VC, vincristine plus cyclophosphamide; VDC, vincristine, doxorubicin, and cyclophosphamide; VIDE, vincristine, ifosfamide, doxorubicin, and etoposide. ${ }^{23}$

the viable component, have relapse rates of nearly $40 \%{ }^{23}$ Based on these data, the SIOP histological risk classification scheme divides renal tumors into three risk categories: low risk, intermediate risk, and high risk (table 3 ). ${ }^{24}$

The SIOP WT2001 trial (table 4) tested the possibility of omitting doxorubicin safely from chemotherapy for stage II/III intermediate-risk WT, after exclusion of a newly defined high risk subgroup (blastemal-type (BT)) from the randomization. Two hundred and ninety-one patients were randomised to actinomycin $\mathrm{D}+$ vincristine + doxorubicin and 292 patients were randomised to actinomycin D+vincristine, with 2-year EFS of (92\% vs $89 \%)$ and 5 -year OS of $96 \%$ in both arms, and in addition to cardiotoxicity that occurred in a $5 \%$ of patients who received doxorubicin, a conclusion comes up that by using stage and histology after neoadjuvant therapy for risk stratification, doxorubicin can 
Table 3 International Society of Pediatric Oncology postoperative histological classification of Wilms' tumor $(\mathrm{WT})^{24}$

\begin{tabular}{lll}
\hline Postoperative histological classification of WT & \\
\hline Low & Intermediate & High \\
Mesoblastic nephroma & WT of epithelial, stromal, & Blastemal-type WT \\
Cystic partially & mixed or regressive types & Diffuse anaplastic WT \\
differentiated & Focal anaplastic WT & Clear cell sarcoma of the \\
Nephroblastoma & & kidney \\
Completely necrotic WT & & Rhabdoid tumor of the \\
& & kidney \\
\hline
\end{tabular}

be safely omitted from management of stage II/III intermediate-risk WT. ${ }^{25}$

BT-WT was identified as a high-risk histological subgroup in WT assessed after pre-nephrectomy chemotherapy in trials of the SIOP Renal Tumor Study Group. Therefore, in SIOP WT2001, postoperative chemotherapy for BT-WT was augmented aiming to survival improvement. BT-WT derived benefits from more intensive chemotherapy as reflected by a reduction in relapse risk. However, the benefit of the more intensive chemotherapy to improve OS was only observed in stage I BT-WTs by adding doxorubicin. ${ }^{26}$

\section{NEUROBLASTOMA (NB)}

Induction phase of chemotherapy in neuroblastoma is of a significant value. Ladenstein and colleagues performed analysis on 549 patients with high-risk neuroblastoma (NB) and confirmed that bone and bone marrow involvement were the only independent adverse prognostic factors. ${ }^{27}$ More recent retrospective studies have shown that metaiodobenzylguanidine (MIBG) response at the end of induction directly correlates with EFS after myeloablative therapy.

In patients with high-risk NB (table 5), ${ }^{28}$ response to induction chemotherapy is emerging as an important determinant of OS. George et al reviewed 43 patients according to specific morphological features. For the majority of patients, induction therapy resulted in a shift from an intermediate/high to low mitosis-karyorrhexis index (MKI) $(p=0.0009)$ and from undifferentiated/poorly differentiated to differentiating tumors $(\mathrm{p}<0.0001)$. Following induction therapy, persistence of intermediate/high tumor MKI and $\geq 90 \%$ persistent neuroblastic cells were predictive of a poor outcome ( $\mathrm{p}=0.001$ and 0.03 , respectively). Less than $10 \%$ tumor necrosis was associated with lower survival. Furthermore, high proliferative activity in the

\begin{tabular}{llll}
\hline $\begin{array}{l}\text { Table } 4 \\
\text { WT2001 }\end{array}$ & Management of Wilms' tumor (WT) according to SIOP \\
\hline \multicolumn{4}{l}{ Management of WT based on risk stratification } \\
\hline \multicolumn{5}{l}{$\begin{array}{l}\text { Stage I } \\
\text { Low risk }\end{array}$} & $\begin{array}{l}\text { No further } \\
\text { management }\end{array}$ & AV & Stage III \\
Intermediate risk & AV & AVD & AV \\
& & $\begin{array}{l}\text { Randomize } \\
\text { AV }\end{array}$ & $\begin{array}{l}\text { AVD/RT } \\
\text { Randomize } \\
\text { AV/RT }\end{array}$ \\
High risk & AVD & High risk+RT & High risk+RT \\
\hline
\end{tabular}

$A V$, actinomycin D+vincristine; $A V D$, actinomycin D+vincristine+doxorubicin; high-risk

chemotherapy, etoposide +carboplatin + cyclophosphamide+doxorubicin ${ }^{25}$; $\mathrm{RT}$, radiotherapy; SIOP, International Society of Pediatric Oncology. primary tumor following induction therapy portends a poor outcome in patients with high-risk NB. A larger cohort study should take place to confirm that tumor histology at second-look surgery could be used to define a subset of very high-risk patients who would benefit from alternative therapies prior to myeloablative dose-intensive transplant. ${ }^{29}$

Another study confirmed that tumor necrosis after neoadjuvant chemotherapy is higher in patients with MYCN amplification. In this study, tumor necrosis after neoadjuvant chemotherapy did not correlate with OS and should not lead to modification of adjuvant chemotherapy. However, these findings need to be confirmed in a larger prospective study of children with high-risk NB. ${ }^{30}$

\section{HEPATOBLASTOMA}

Hepatoblastoma is the most common hepatic malignancy in children. ${ }^{31}$ Children's Oncology Group stages III and IV usually have surgically unresectable tumors and require neoadjuvant chemotherapy. ${ }^{32}$ Five-year EFS of greater than $90 \%$ is seen in patients with tumors that were surgically removed at diagnosis, whereas 5-year EFS rates of approximately $64 \%$ for stage III patients and 25\% for stage IV patients who had unresectable tumors. ${ }^{31}$

Histopathological changes in hepatoblastoma after chemotherapy, including tumor necrosis, have been previously described. ${ }^{33-35}$ Wang et al described specific necrosis and fibrohistiocytic response following neoadjuvant therapy in 22 patients with hepatoblastoma, and besides having characteristic necrosis and fibrohistiocytic response, two-thirds had areas of cytoarchitectural differentiation mimicking non-neoplastic liver, and a quarter had alterations mimicking hepatocellular carcinoma. They also concluded that multifocality, greater post-treatment necrosis and hepatocellular carcinoma-like morphology were more often associated with metastatic disease. ${ }^{33}$ In a study of 17 patients with hepatoblastoma, the extent of tumor necrosis was associated with better outcome. ${ }^{35}$ In their study, Venkatramani et al revealed that the risk of disease progression/ relapse and the risk of death in hepatoblastoma decreased significantly with increasing percentage of tumor necrosis. They used the 30\% cut-off for tumor necrosis for this study that could differ in studies with larger sample sizes. They concluded that the low cut-off of 30\% tumor necrosis was predictive of survival (figure 7), adding that the extent of tumor necrosis following neoadjuvant chemotherapy is an independent prognostic factor in patients with newly diagnosed hepatoblastoma and that histological response might be used in strategies to modify adjuvant chemotherapy to improve survival in hepatoblastoma. ${ }^{36}$

\section{RHABDOMYOSARCOMA (RMS)}

Surgery constitutes a major role in treating rhabdomyosarcoma (RMS) from initial planned biopsy till attempting total tumor excision. Diagnosis, management and late effects depend on careful planning and execution of biopsy. The earliest classification system for RMS, the Intergroup Rhabdomyosarcoma Study (IRS) clinical grouping system, is based on the amount of tumor remaining after the first surgical intervention (table 6). ${ }^{8}$

Tumor necrosis and histological changes in tumor post neoadjuvant chemotherapy was not a part of any RMS 
Table 5 Children's Oncology Group neuroblastoma (NB) risk stratification system ${ }^{28}$

\begin{tabular}{|c|c|c|c|c|c|}
\hline \multicolumn{6}{|c|}{ Children's Oncology Group NB risk stratification system } \\
\hline INSS* stage & Age & MYCN status & Shimada & DNA index & Risk group \\
\hline 1 & $0-21$ years & Any & Any & Any & Low \\
\hline \multirow[t]{4}{*}{$2 \mathrm{~A} / 2 \mathrm{~B}$} & $<365$ days & Any & Any & Any & Low \\
\hline & $\geq 365$ days -21 years & Normal & Any & - & Low \\
\hline & $\geq 365$ days -21 years & Amplified & Favorable & - & Low \\
\hline & $\geq 365$ days -21 years & Amplified & Unfavorable & - & High \\
\hline \multirow[t]{5}{*}{3} & $<365$ days & Normal & Any & Any & Intermediate \\
\hline & $<365$ days & Amplified & Any & Any & High \\
\hline & $\geq 365$ days -21 years & Normal & Favorable & - & Intermediate \\
\hline & $\geq 365$ days -21 years & Normal & Unfavorable & - & High \\
\hline & $\geq 365$ days -21 years & Amplified & Any & - & High \\
\hline \multirow[t]{3}{*}{4} & $<365$ days & Normal & Any & Any & Intermediate \\
\hline & $<365$ days & Amplified & Any & Any & High \\
\hline & $\geq 365$ days -21 years & Any & Any & - & High \\
\hline \multirow[t]{4}{*}{$4 \mathrm{~S}$} & $<365$ days & Normal & Favorable & $>1$ & Low \\
\hline & $<365$ days & Normal & Any & $=1$ & Intermediate \\
\hline & $<365$ days & Normal & Unfavorable & Any & Intermediate \\
\hline & $<365$ days & Amplified & Any & Any & High \\
\hline
\end{tabular}

*INSS, International Neuroblastoma Staging System

protocol and have not been studied in this perspective despite some studies that described treatment-related changes that occurred in RMS. Some reports stated that
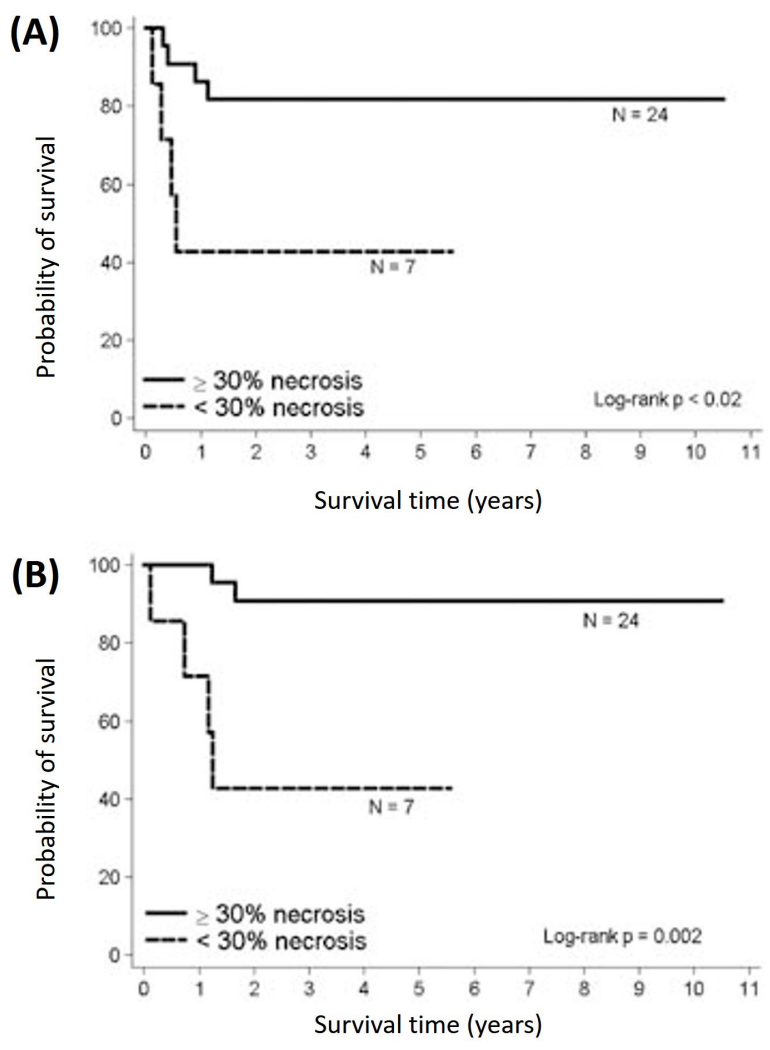

Figure 7 Kaplan-Meier curves of hepatoblastoma (HB) patients with $<30 \%$ tumor necrosis and $\geq 30 \%$ necrosis following neoadjuvant chemotherapy. (A) Event-free survival and (B) overall survival. $^{36}$
Table 6 Intergroup Rhabdomyosarcoma Study Group (IRSG) surgical-pathological grouping system of rhabdomyosarcoma (RMS) ${ }^{8}$

\begin{tabular}{|c|c|}
\hline \multicolumn{2}{|c|}{ IRSG surgical-pathological grouping system of RMS } \\
\hline Group & Definition \\
\hline I & $\begin{array}{l}\text { Localized tumor, completely removed with pathologically clear } \\
\text { margins and no regional lymph node involvement }\end{array}$ \\
\hline II & $\begin{array}{l}\text { Localized tumor, grossly removed with (a) microscopically involved } \\
\text { margins, (b) involved, grossly resected regional lymph nodes, or (c) } \\
\text { both }\end{array}$ \\
\hline III & $\begin{array}{l}\text { Localized tumor, with gross residual disease after grossly } \\
\text { incomplete removal, or biopsy only }\end{array}$ \\
\hline IV & Distant metastases present at diagnosis \\
\hline
\end{tabular}

surgical or pathological findings were always corresponding to the imaging appearance of RMS. Poor radiological response or residual large mass on CT scanning may not represent persistent viable tumor and might instead be due to reactive or reparative changes, cytodifferentiation, necrosis, inflammation, and fibrosis. ${ }^{37} 38$ Furthermore, post chemotherapy samples can show a spectrum of necrosis; inflammation with macrophages, lymphocytes, and plasma cells; fibrosis; and atrophic or regenerating non-neoplastic skeletal muscle.

Moreover, it was noticed that in patients with gross residual tumor when chemotherapy begins (groups III and IV), second-look operations and delayed excision of residual tumor masses, even after radiation therapy and chemotherapy, may improve the OS. ${ }^{8}$ In a retrospective study of second-look operations in IRS-III, $12 \%$ of patients clinically believed to have had a complete response had residual viable tumor and three-fourths of patients clinically believed to have had a partial response or no response either had no viable tumor or were converted to a complete response at surgery. These converted patients had the same 3 -year survival as those whose complete responses were 
confirmed at second-look surgery $(73 \%$ and $80 \%$, respectively). ${ }^{39}$ These findings confirm that detection of viable tumor cells in tumor samples might be essential to direct further management in RMS. Another single-institution study of 48 IRS- III patients showed that those who had a delayed complete surgical excision $(n=22)$ had superior relapse free survival RFS and OS to those who did not (approximately $90 \%$ vs $60 \%$ RFS, p=0.013). However, patient numbers were too small to permit a multivariate analysis, but histology and nodal status were also significant variables in univariate analysis. ${ }^{40}$

This area of study with regard to tumor changes in RMS post chemotherapy and a complete understanding of the clinical relevance of what the results could be necessitate further studies.

\section{CONCLUSION}

Tumor necrosis and histopathological changes in childhood solid tumors following neoadjuvant chemotherapy are important predictors of disease outcome. There are better OS, EFS and even lower rate of local recurrence in good responders with high tumor necrosis. However, there is little evidence to recommend for more intensified adjuvant chemotherapy regimens for poor responders. Toxicities from intensified adjuvant chemotherapy and comparable disease outcome are among the main causes behind these results. Trends toward intensifying neoadjuvant chemotherapy for better histopathological responses or addition of newer chemotherapeutic agent to the adjuvant arm should be a subject to more large trials in order to use the beneficial value of tumor histopathological changes as an important predictor of disease outcome.

Correction notice This article has been corrected since it was published Online First. Figure 5 has been replaced with the correct figure.

Acknowledgements We gratefully thank Dr Omar Al Zahrani, director of Prince Sultan Oncology Center for his support and continuous encouragement of research development at the center.

Contributors EH wrote the article, reviewed it and completed all necessary requirements. AAJ and MAP have substantial contributions to conception and design, revising it critically for important intellectual content and final approval of the version to be published. AD, FN and GG have substantial contributions to conception and design, acquisition of the data and drafting the article.

\section{Competing interests None declared.}

Provenance and peer review Not commissioned; externally peer reviewed.

Open Access This is an Open Access article distributed in accordance with the Creative Commons Attribution Non Commercial (CC BY-NC 4.0) license, which permits others to distribute, remix, adapt, build upon this work noncommercially, and license their derivative works on different terms, provided the original work is properly cited and the use is non-commercial. See: http:// creativecommons.org/licenses/by-nc/4.0/

(C) American Federation for Medical Research (unless otherwise stated in the text of the article) 2018. All rights reserved. No commercial use is permitted unless otherwise expressly granted.

\section{REFERENCES}

1 Huvos AG. Bone tumors: diagnosis, treatment, and prognosis. 2nd edition. Philadelphia, PA, USA: W.B. Saunders, 1990.

2 Wunder JS, Paulian G, Huvos AG, et al. The histological response to chemotherapy as a predictor of the oncological outcome of operative treatment of Ewing sarcoma. J Bone Joint Surg Am 1998;80:1020-33.

3 Jaffe N. Chemotherapy for malignant bone tumors. Orthop Clin North Am $1989: 20: 487-503$.
4 Picci P, Rougraff BT, Bacci G, et al. Prognostic significance of histopathologic response to chemotherapy in nonmetastatic Ewing's sarcoma of the extremities. J Clin Oncol 1993;11:1763-9.

5 Picci P, Böhling T, Bacci G, et al. Chemotherapy-induced tumor necrosis as a prognostic factor in localized Ewing's sarcoma of the extremities. J Clin Oncol 1997;15:1553-9.

6 Rosen G, Murphy ML, Huvos AG, et al. Chemotherapy, en bloc resection, and prosthetic bone replacement in the treatment of osteogenic sarcoma. Cancer 1976;37:1-11.

7 Rosen G, Marcove RC, Caparros B, et al. Primary osteogenic sarcoma: the rationale for preoperative chemotherapy and delayed surgery. Cancer 1979;43:2163-77

8 Stuart H, David E, Thomas L, et al; Nathan and Oski's hematology and oncology of infancy and childhood. Eighth edition.

9 Goorin AM, Schwartzentruber DJ, Devidas M, et al. Presurgical chemotherapy compared with immediate surgery and adjuvant chemotherapy for nonmetastatic osteosarcoma: Pediatric Oncology Group Study POG-8651. J Clin Oncol 2003;21:1574-80.

10 Winkler K, Beron G, Kotz R, et al. Neoadjuvant chemotherapy for osteogenic sarcoma: results of a Cooperative German/Austrian study. J Clin Oncol 1984;2:617-24.

11 Bacci G, Picci P, Ruggieri P, et al. Primary chemotherapy and delayed surgery (neoadjuvant chemotherapy) for osteosarcoma of the extremities. The Istituto Rizzoli Experience in 127 patients treated preoperatively with intravenous methotrexate (high versus moderate doses) and intraarterial cisplatin. Cancer 1990;65:2539-53.

12 Winkler K, Beron G, Delling G, et al. Neoadjuvant chemotherapy of osteosarcoma: results of a randomized cooperative trial (COSS-82) with salvage chemotherapy based on histological tumor response. J Clin Oncol 1988;6:329-37.

13 Bacci G, Ferrari S, Bertoni F, et al. Long-term outcome for patients with nonmetastatic osteosarcoma of the extremity treated at the istituto ortopedico rizzoli according to the istituto ortopedico rizzoli/osteosarcoma-2 protocol: an updated report. J Clin Oncol 2000; 18:4016-27.

14 Smeland S, Müller C, Alvegard TA, et al. Scandinavian Sarcoma Group Osteosarcoma Study SSG VIII: prognostic factors for outcome and the role of replacement salvage chemotherapy for poor histological responders. Eur J Cancer 2003;39:488-94

15 Marina NM, Smeland S, Bielack SS, et al. Comparison of MAPIE versus MAP in patients with a poor response to preoperative chemotherapy for newly diagnosed high-grade osteosarcoma (EURAMOS-1): an open-label, international, randomised controlled trial. Lancet Oncol 2016;17:1396-408.

16 Lin PP, Jaffe N, Herzog CE, et al. Chemotherapy response is an important predictor of local recurrence in Ewing sarcoma. Cancer 2007;109:603-11.

17 Essell JH, Schroeder MT, Harman GS, et al. Ursodiol prophylaxis against hepatic complications of allogeneic bone marrow transplantation. A randomized, double-blind, placebo-controlled trial. Ann Intern Med 1998:128:975-81.

18 Smith MA, Ungerleider RS, Horowitz ME, et al. Influence of doxorubicin dose intensity on response and outcome for patients with osteogenic sarcoma and Ewing's sarcoma. J Natl Cancer Inst 1991;83:1460-70.

19 Whelan J, Deley M-CL, Dirksen U, et al. Efficacy of busulfan-melphalan high dose chemotherapy consolidation (BuMel) in localized high-risk Ewing sarcoma (ES): Results of EURO-EWING 99-R2 randomized trial (EE99R2Loc). J Clin Oncol 2016;34.

20 Gaspar N, Hawkins DS, Dirksen U, et al. Ewing Sarcoma: Current Management and Future Approaches Through Collaboration. J Clin Oncol 2015;33:3036-46.

21 Green DM. The treatment of stages I-IV favorable histology Wilms' tumor. J Clin Oncol 2004;22:1366-72.

22 Boccon-Gibod L, Rey A, Sandstedt B, et al. Complete necrosis induced by preoperative chemotherapy in Wilms tumor as an indicator of low risk: report of the international society of paediatric oncology (SIOP) nephroblastoma trial and study 9. Med Pediatr Oncol 2000;34:183-90.

23 Weirich A, Leuschner I, Harms D, et al. Clinical impact of histologic subtypes in localized non-anaplastic nephroblastoma treated according to the trial and study SIOP-9/GPOH. Ann Oncol 2001;12:311-9.

24 Vujanić GM, Sandstedt B, Harms D, et al. Revised International Society of Paediatric Oncology (SIOP) working classification of renal tumors of childhood. Med Pediatr Oncol 2002;38:79-82.

25 43rd Congress of the International Society of Paediatric Oncology (SIOP) 2011. SIOP Abstracts. Pediatr blood cancer 2011. 57. Auckland,New Zealand, 2011:705-897.

26 van den Heuvel-Eibrink MM, van Tinteren $\mathrm{H}$, Bergeron C, et al. Outcome of localised blastemal-type Wilms tumour patients treated according to intensified treatment in the SIOP WT 2001 protocol, a report of the SIOP Renal Tumour Study Group (SIOP-RTSG). Eur J Cancer 2015;51:498-506. 
27 Ladenstein R, Philip T, Lasset C, et al. Multivariate analysis of risk factors in stage 4 neuroblastoma patients over the age of one year treated with megatherapy and stem-cell transplantation: a report from the European Bone Marrow Transplantation Solid Tumor Registry. J Clin Oncol 1998;16:953-65.

28 Weinstein JL, Katzenstein HM, Cohn SL. Advances in the diagnosis and treatment of neuroblastoma. Oncologist 2003;8:278-92.

29 George RE, Perez-Atayde AR, Yao X, et al. Tumor histology during induction therapy in patients with high-risk neuroblastoma. Pediatr Blood Cancer 2012:59:506-10.

30 Bomken S, Davies B, Chong L, et al. Percentage tumor necrosis following chemotherapy in neuroblastoma correlates with MYCN status but not survival. Pediatr Hematol Oncol 2011;28:106-14.

31 Stiller CA, Pritchard J, Steliarova-Foucher E. Liver cancer in European children: incidence and survival, 1978-1997. Report from the Automated Childhood Cancer Information System project. Eur J Cancer 2006;42:2115-23.

32 Ortega JA, Douglass EC, Feusner JH, et al. Randomized comparison of cisplatin/vincristine/fluorouracil and cisplatin/continuous infusion doxorubicin for treatment of pediatric hepatoblastoma: A report from the Children's Cancer Group and the Pediatric Oncology Group. J Clin Oncol 2000;18:2665-75.
33 Wang LL, Filippi RZ, Zurakowski D, et al. Effects of neoadjuvant chemotherapy on hepatoblastoma: a morphologic and immunohistochemical study. Am I Surg Pathol 2010;34:287-99.

34 Saxena R, Leake JL, Shafford EA, et al. Chemotherapy effects on hepatoblastoma. A histological study. Am J Surg Pathol 1993;17:1266-71.

35 Heifetz SA, French M, Correa M, et al. Hepatoblastoma: the Indiana experience with preoperative chemotherapy for inoperable tumors; clinicopathological considerations. Pediatr Pathol Lab Med 1997;17:857-74.

36 Venkatramani R, Wang L, Malvar J, et al. Tumor necrosis predicts survival following neo-adjuvant chemotherapy for hepatoblastoma. Pediatr Blood Cancer 2012;59:493-8.

37 Lowichik A, Zhou H, Pysher TJ, et al. Therapy associated changes in childhood tumors. Adv Anat Pathol 2000;7:341-59.

38 Atra A, Ward HC, Aitken K, et al. Conservative surgery in multimodal therapy for pelvic rhabdomyosarcoma in children. Br I Cancer 1994;70:1004-8.

39 Breneman JC, Wiener ES. Issues in the local control of rhabdomyosarcoma. Med Pediatr Oncol 2000;35:104-9.

40 Viswanathan AN, Grier HE, Litman HJ, et al. Outcome for children with group III rhabdomyosarcoma treated with or without radiotherapy. Int I Radiat Oncol Biol Phys 2004;58:1208-14. 